\title{
HIGH POWER COUPLER STUDIES FOR THE ERLP
}

\author{
J. Rogers, C. Beard, P. Corlett, \\ ASTeC, CCLRC Daresbury Laboratory, Warrington, UK, WA4 4AD
}

\section{Abstract}

Two Superconducting RF modules of the ELBE type have been ordered from Accel Instruments $\mathrm{GmbH}$ for use on the Energy Recovery Linac Prototype (ERLP) being built at Daresbury Laboratory. One structure is to be used as a booster module, with an energy gain of $8 \mathrm{MeV}$, and the other is to act as an energy recovery linac operating at electron beam energy of up to $35 \mathrm{MeV}$. High power couplers capable of handling up to $10 \mathrm{~kW} \mathrm{CW}$ are required to provide successful operation of the ERLP.

Once received from Accel four couplers including RF windows will be conditioned and tested at FZR Dresden; this paper describes the test procedure anticipated.

\section{RF WINDOWS}

$\mathrm{RF}$ power is fed from the source to the test stand using WR650 waveguide. A circulator is used to protect the source from any reflected power due to mismatches in the system during the conditioning process (see figure 1 below). The reflected power is dissipated in a water cooled load that has to be able to withstand total reflection. A directional coupler is inserted in this section of waveguide enabling the measurement of forward and reflected power.

The first window is attached to the flange of an $\mathrm{H}$ plane bend. After this window the bend must be maintained at vacuum conditions with a pressure less than $5 \times 10^{-7}$ mBar. The $\mathrm{H}$ - plane bend is $1 / 4$ guided wavelengths $\left(\lambda_{\mathrm{g}}\right)$ long at $1.3 \mathrm{GHz}$ (see figure 2).

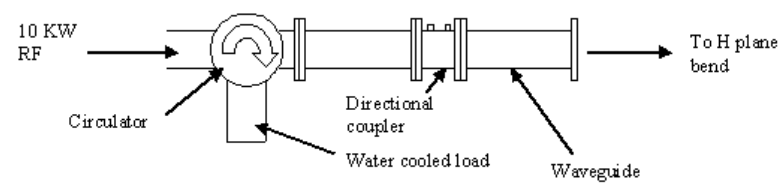

Figure 1: Waveguide system.

A photo multiplier tube is used to detect the emission of light from any incidents of arcing (a form of voltage breakdown). A photo multiplier is inserted adjacent to the RF window to detect any breakdown and is used to ensure full conditioning of the component (see figure 3 ). An infrared sensor is employed to monitor the temperature of the window throughout the process. A straight section of waveguide $1 \frac{1}{4} \lambda_{\mathrm{g}}$ in length is attached after the detector section; this has a shorting plate attached to the end flange. The shorting plate causes total reflection of the RF power back towards the second window resulting in the formation of a standing wave. The first window is set to an electric field minimum of the standing wave and the second window is set at a maximum. This high electric field conditions the second window.

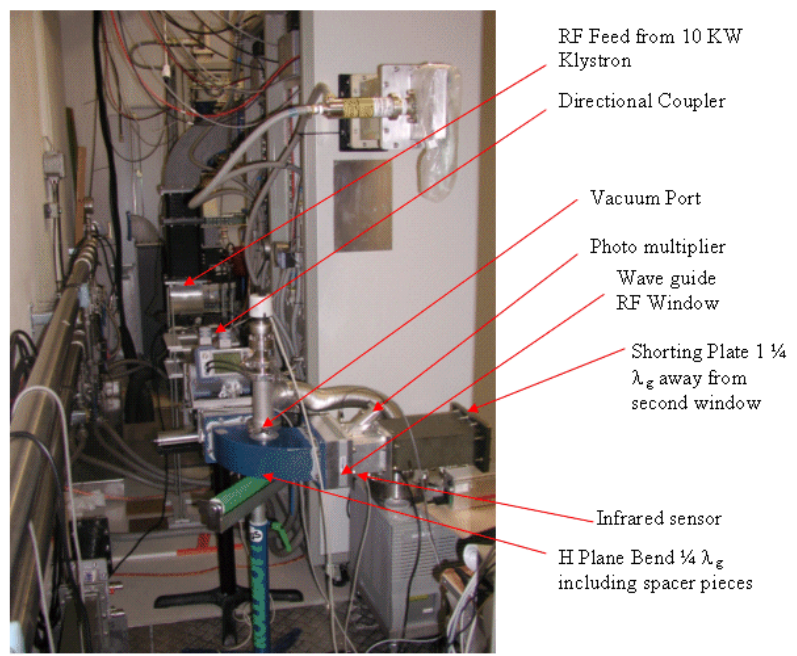

Figure 2: Waveguide window test apparatus.

The forward and reflected power, photo multiplier tubes, temperature sensors and vacuum gauges are all monitored and form interlocks using a crate of electronic equipment which interfaces with a PC. The PC is used to run the software, which automatically conditions and tests the waveguide windows as well as the couplers. All interlocks are fail safe and are not solely reliant upon the $\mathrm{PC}[1]$.

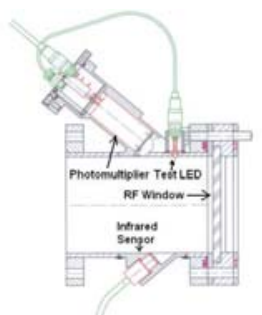

Figure 3: Detail view of waveguide window assembly.

\section{Window Testing Procedure}

- Windows cleaned

- Components assembled in clean room conditions

- Waveguide evacuated to $\approx 2 \times 10^{-6} \mathrm{mBar}$

- Software used to control test stand: User defined RF Pulse typically $10 \mathrm{mS}$ duration with a repetition rate of $300 \mathrm{mS}$. After 300 successful RF pulses power increased by a further 100 Watts.

- Program ends after 300 Pulses @ 10 KW RF power have been applied

- Conditioning can take between 6 hours and 2 days 


\section{RF COUPLERS}

The RF power is supplied via WR650 waveguide. The power is transferred to the coupler by a waveguide to coaxial transition known as a door knob (see figure 4). Under test conditions the door knob section is not under vacuum. Once in service the coupler assembly would be subject to vacuum and cryogenic conditions.

The door knob transition contains a photo multiplier tube to detect the emission of light from arcing events. This is most likely to occur in the door knob transition and the cold window sections due to the high electric field present in these areas (see figure 5). A PT100 temperature sensor is connected to the inner conductor on the cold section for temperature measurements during testing. The temperature should be maintained below $70^{\circ} \mathrm{C}$ through out the operation.

The coupler assembly is bolted to the flange on the test cavity. The antenna penetrates into the cavity section exciting the electric field. The cavity and the cold section are maintained at vacuum conditions in excess of $5 \times 10^{-4}$ mBars.

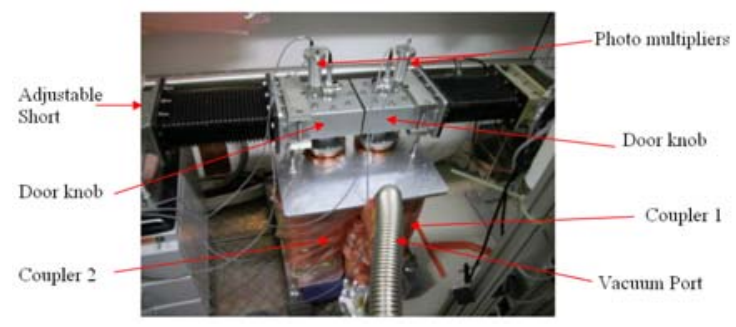

Figure 4: Coupler test stand.

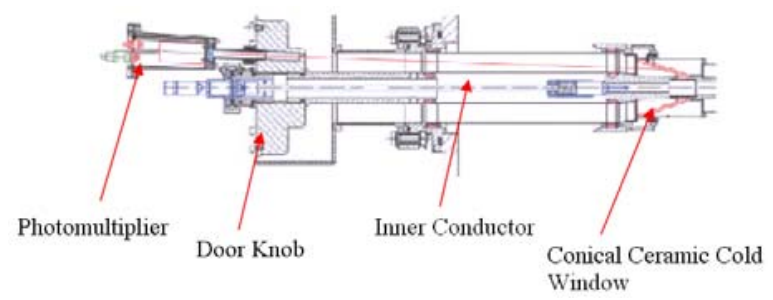

Figure 5: Drawing of coupler and door knob transition.

\section{Coupler Testing Procedure}

- Coupler Components assembled in clean room

- Mount couplers into test stand cavity

- Evacuate test stand and coupler

- Run coupler conditioning software as before

- Program ends after 300 RF pulses @ 10 KW have been successfully applied

The dimensions of the test cavity should be such that it is resonant at the desired operating frequency of the couplers $(1.3 \mathrm{GHz})$ to allow maximum transmission through the set-up. The power within the test waveguide is coupled out of the cavity by the insertion of coupler 2 . A precision adjustable short is attached to the waveguide flange after coupler 2. This enables the phase of the standing wave to be adjusted ensuring that all areas of the couplers are exposed to the maximum electric field to provide the best environment for conditioning.

\section{Coupler Limitations}

At present the couplers have only been tested up to a maximum of $10 \mathrm{~kW}$ continuous wave. In order to be able to operate the ERLP at higher powers, a full understanding of the coupler limitations is required.

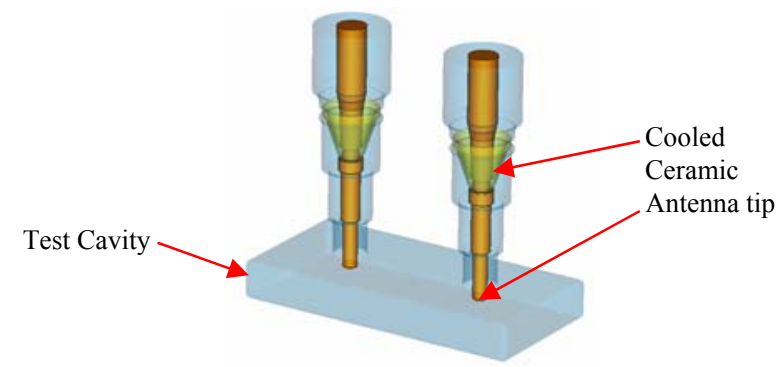

Figure 6: Simulation of the couplers and test stand.

Using Microwave Studio we have been able to recognise the areas for concern for this particular design. It has also been possible to address one of the problems with the test stand being used. This showed an offfrequency cavity being used, requiring the couplers to be switched to allow the second to be conditioned.

The couplers are tested in a back-to-back arrangement (see figure 6). This requires one coupler to act as a transmitter and the other coupler to act as a receiver.

\section{Voltage Breakdown}

From the simulations two regions were observed to be of concern for thermal breakdown due to the high field stresses. One area is around the conical shaped ceramic window (see figure 7). This area of the ceramic however is cooled to liquid nitrogen $\left(\mathrm{LN}_{2}\right)$ temperatures. The thermal gradient across the ceramic can be tolerated. This is due to the material properties of the ceramic that leads to excellent thermal conduction at $\mathrm{LN}_{2}$ temperatures.
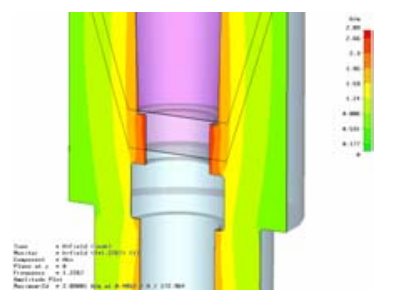

Figure 7: Peak fields on the conical ceramic.

The other region for concern is located around the antenna tip (see figure 8). The conduction of the heat dissipated in the antenna tip results in a significant temperature rise in the tip. This leads to radiative heating of the structures beam pipe. The temperature is maintained well below the level that would cause thermal damage. Thermal contact between the room temperature section and $\mathrm{LN}_{2}$ cooled coax section is only through the finger stock contacts. 


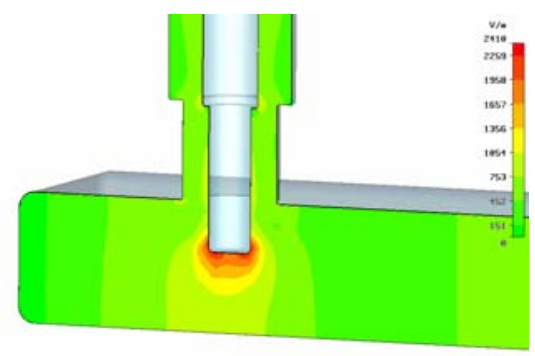

Figure 8: Peak fields on the antenna tip.

At present the maximum that the couplers can be tested to is $10 \mathrm{~kW} \mathrm{CW}$. In order to be able to test the breakdown limit of the coupler design we are investing in equipment to build a resonant ring, which will allow higher fields to be achieved with the same input power. This structure also operates in the travelling wave mode which would allow a full sweep of the coupler to ensure optimum conditioning.

\section{RESONANT RING}

A resonant ring is a device consisting of a loop of transmission line (typically waveguide). This loop is stimulated with RF power by means of a directional coupler.

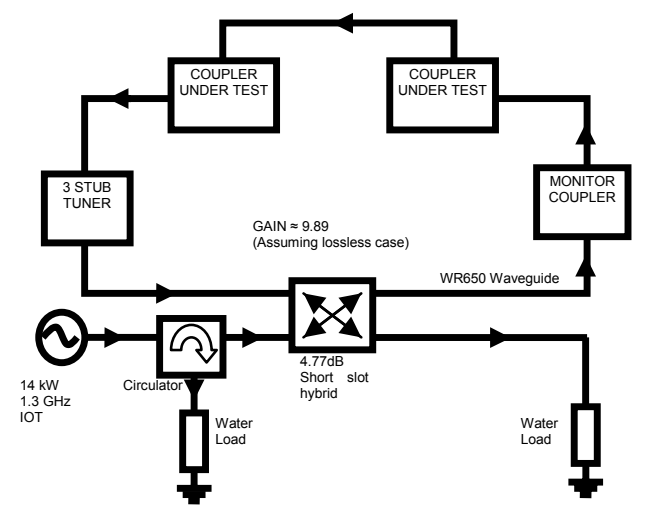

Figure 9: Resonant ring schematic.

If the path length of the ring is equal to an integer number of RF wavelengths, the power coupled into the ring will be in phase with the power already circulating in the ring, thus they add constructively.

This results in an accumulation of power within the ring until an equilibrium condition is reached where the power coupled back out of the ring is equal to that of the initial power source, this power is then dissipated in the matched load.

At this equilibrium condition, the RF power circulating within the ring can be significantly higher than the power of the initial RF source.
By using these high circulating powers, passive, non dissipative components such as RF windows and couplers can be tested, without the expense and inconvenience of using a full power test set up (see figure 9).

\section{Limitations}

There are 3 factors which define the gain of a resonant ring, these are:

- Coupling factor of the directional coupler

- The attenuation within the ring

- The reflection coefficient of the ring

The attenuation is a physical property of the ring relating to the construction and materials of the ring, the only parameter that can be easily controlled is the coupling factor and the reflection.

The gain of the ring is given by the following equation.

$$
G=C^{2}\left(\frac{1-e^{-\alpha L} \sqrt{1-C^{2}} \sqrt{1-r^{2}}}{1-2 e^{-\alpha L} \sqrt{1-C^{2}} \sqrt{1-r^{2}}+e^{-2 \alpha L}\left(1-C^{2}\right)}\right)^{2}
$$

Where $e^{-\alpha L}=$ Ring attenuation

$r=$ Reflection Coefficient

$C=$ Coupling factor of hybrid

Reflection can be effectively removed by impedance matching and altering coupler parameters, and so can be ignored. Therefore (1) can be simplified to:

$$
G=\frac{C^{2}}{\left|1-T \sqrt{1-C^{2}}\right|^{2}}
$$

\section{Where $T=$ Transmission coefficient}

The full derivation of equations (1) and (2) above can be found in references [2] and [3].

The Resonant ring proposed for Daresbury Laboratory will use a signal source of $1.3 \mathrm{GHz}$ at up to $14 \mathrm{~kW} \mathrm{CW}$. The couplers will be tested to a power of $50 \mathrm{~kW}$.

\section{REFERENCES}

[1] A. Buchner, H. Buttig, J. Stephen, FZR Dresden, Germany,."RF window diagnosis and training for the ELBE superconducting accelerator", Workshop on high power couplers for superconducting accelerators, Jefferson Lab, Newport News, Virginia, October 30 2002.

[2] V. Veshcherevich, "Resonant ring for high power tests of RF couplers" Cornell University Ithaca, NY 14853.

[3] E. Gerken, J.M. Gahl, D. Rees, W. Roybal, K Cummings, "Resonant Ring for Testing of Accelerator RF Windows", PAC 12-16 May 1997, Vancouver, BC, Canada. 